\title{
La comunicación organizacional en las Entidades Deportivas Náuticas. Propuesta de modelo estratégico y relacional \\ Organizational communication in nautical sports entities. Proposal of a strategic and relational model \\ *Juan Monserrat-Gauchi, **Paula González-Redondo, *Concepción Campillo-Alhama \\ *Universidad de Alicante (España), **Instituto Mediterráneo de Estudios de Protocolo (España)
}

\begin{abstract}
Resumen. Se realiza un análisis descriptivo de la gestión de la comunicación en las organizaciones deportivas náuticas en España desde los parámetros de las teorías estratégicas de la comunicación alineadas en la perspectiva relacional. El objetivo es doble: la realización de dicho análisis descriptivo en estas entidades y proporcionar un modelo de gestión de la comunicación válido para las mismas. Se toma como universo de estudio la totalidad de entidades náuticas deportivas inscritas en la Real Federación Española de Vela. La metodología supone la elaboración de un cuestionario como herramienta para el análisis. Los resultados muestran que no se lleva a cabo una planificación estratégica de la comunicación con respecto a sus públicos, una gestión anclada en modelos bidireccionales y pocos recursos humanos destinados a la planificación y gestión de la comunicación. En definitiva, este tipo de organización deportiva no se ha adaptado a las necesidades actuales. El artículo propone un modelo estratégico de gestión de la comunicación para este tipo de entidades. Palabras Clave: comunicación-deportiva, comunicación-organizacional, comunicación-estratégica, gestión-relacional, entidades deportivas náuticas, nueva teoría estratégica, plan estratégico de comunicación.
\end{abstract}

\begin{abstract}
Adescriptive analysis of the management of communication in nautical sports organizations in Spain is carried out. This is done using the parameters of the strategic theories of communication aligned with the relational perspective. The objective is twofold: to carry out a descriptive analysis in these organisations, and to provide a valid communication management model for them. The population universe comprised all nautical sports entities registered in the Royal Spanish Sailing Federation. The methodology includes the development of a questionnaire as a tool for the analysis. The results show that there is no strategic planning of communications regarding targets, as well as their management is deeply rooted in bidirectional models. Moreover, few human resources are destined to the planning and management of communication. In conclusion, this type of sports organizations have not adapted to what their needs are nowadays. The article proposes a strategic communication management model for this type of entities.

Keywords: sports communication, organizational communication, strategic communication, relational management, nautical sports entities, new strategic theory, strategic communication plan.
\end{abstract}

\section{Introducción}

A día de hoy, los procesos comunicativos y relacionales de cualquier tipo de empresa, institución o entidad han cambiado en gran parte debido a la aparición del medio internet (Túñez-López, 2012; Serrano-Cobos, 2016; VilaplanaAparicio, Iglesias-García \& Martín-Llaguno, 2018; Masip, Ruiz-Caballero \& Suau, 2019). Estos cambios también se han producido en las entidades deportivas náuticas (en adelante: EDN), sujeto de estudio en este artículo, que tendrán que implantar un modelo de comunicación y gestión relacional planificada y gestionada bajo el enfoque de una comunicación integral.

La planificación de la estrategia permite aproximar la profesión comunicativa, tradicionalmente sometida a críticas por su carácter inesperado y fortuito, al ámbito objetivo de la investigación y la sistematización (Segarra-Saavedra, Martínez-Sala \& Monserrat-Gauchi, 2018). No obstante, todavía en algunos ámbitos profesionales, la planificación de la estrategia es sobreentendida como la mera relación con los medios de comunicación.

Así, respecto a las EDN existen autores que manifiestan que el gabinete de comunicación tiene como finalidad la comunicación del club con los medios de comunicación, crear noticias positivas y estar pendiente de los periodistas críti-

Fecha recepción: 28-02-19. Fecha de aceptación: 26-04-20

Juan Monserrat-Gauchi

Juan.Monserrat@ua.es cos con la gestión del mismo (Olabe-Sánchez, 2015). No obstante, defienden que debe asumir responsabilidades vinculadas con la planificación de la comunicación desde una perspectiva relacional en base a sus públicos (Olabe- Sánchez, 2009; García-Orosa, 2005; Fernández-Souto, Puentes-Rivera \& Vázquez-Gestal, 2019; Alguacil, Crespo-Hervás \& PérezCampos 2020). Los autores de esta investigación se alinean con la corriente que concibe la planificación de la estrategia de comunicación como una labor que va más allá de las meras relaciones con los medios e incluso con sus públicos. De esta manera adquiere el rigor requerido, recurrentemente demandado por todos los agentes implicados en el mercado y por la comunidad académica desde diferentes perspectivas profesionales (Soler-Adillon, Sora, Freixa \& Ribas, 2016; Segarra-Saavedra, et al., 2018; Carrillo-Durán, 2016; TúñezLópez, 2012).

El presente trabajo intenta contribuir a esta perspectiva al aportar una propuesta práctica de sistematización de la planificación de la comunicación, que están desarrollando diferentes autores enmarcados en la denominada Nueva Teoría Estratégica (NTE), (Pérez-González \& Massoni, 2009). Esta nueva teoría, lleva años intentando sistematizar metodologías de planificación válidas en el ámbito profesional.

\section{La Planificación Estratégica de la comunicación des-} de una perspectiva relacional

En el ámbito de la comunicación el escenario ha cambiado notablemente desde los años 90. Por un lado, la aparición de Internet como medio que concede voz y participación a 
los públicos de empresas e instituciones y, por otro lado, los cambios sociales como familias monoparentales, aumento de personas solteras, disminución de la natalidad, etc. (INE, 2018). Las fuentes e investigaciones académicas se hacen eco de estos cambios, y por tanto, reflejan nuevas conceptualizaciones que la comunicación ha de comprender y asumir si desea ser eficaz. Éstas provienen desde distintas perspectivas: sociedad líquida (Bauman, 2013), sociedad red (Castells, 2000), postpublicidad (Solana, 2010), o funcionamiento social en cardumen (Ortiz, 2010), entre otros.

Uno de los principales cambios producido entre los agentes de la comunicación es el llevado a cabo por los antaño denominados consumidores, individuos, públicos o audiencias de las empresas o de los medios (fueran compradores o no de sus productos/servicios) (Masip, et al., 2019). Éstos han pasado de ser meros espectadores de la realidad que les envolvía a ser sujetos activos, creadores o co-creadores de contenidos comunicativos (prosumidores o prosumers) (Martínez-Sala, Segarra-Saavedra \& Monserrat-Gauchi, 2018)

El cambio en este agente de la comunicación, conlleva inevitablemente la necesidad de adaptación de los demás (instituciones, empresas, agencias de comunicación) para seguir siendo eficaces. Por tanto, se hace necesaria la integración de perfiles profesionales especializados como el social media strategist, content manager, content curator, record manager y community manager, en el seno de empresas e instituciones (Soler-Adillon, et al., 2016; CarrilloDurán, 2016) para logar una correcta gestión relacional de las marcas. Esta perspectiva va más allá de la mera relación con los medios de comunicación, los cuales, continuarán siendo uno de los públicos a considerar. Sólo con la correcta planificación y gestión de la comunicación se potenciará el papel de los consumidores y público en general, como prosumers y adprosumers (Martínez-Sala, et al., 2018) a partir del establecimiento de vínculos emocionales con todos sus públicos. Estos son clave en la supervivencia de un tipo de organización sin ánimo de lucro como las EDN.

El informe Social Media Marketing Industry Report, con respecto al comportamiento global de empresas e instituciones, manifiesta que un $92 \%$ de empresas asumen la importancia de las redes sociales para comercializar sus marcas y productos. Y lo hacen gracias a su capacidad para aumentar la notoriedad de la marca, lograr fidelización y como un valioso recurso para obtener información de mercado (Stelzner, 2017).

La vertiente relacional de la estrategia de comunicación, supone asumir que los consumidores y los públicos son los verdaderos portavoces poderosos de la marca, ya que adoptan el papel de adprosumers (Caro, Luque \& Zayas, 2015; Marauri-Castillo, Pérez-Dasilva \& Rodríguez-González, 2015; Martínez-Sala \& Campillo-Alhama, 2018). Ésta es una realidad ya asumida tanto a nivel académico como profesional. Se trata de constatar si las EDN la han integrado en la gestión de su comunicación.

Kotler, reivindica la evolución del marketing transaccional al relacional desde 2004. Esta perspectiva relacional posee como finalidad principal establecer relaciones con sus consumidores para fidelizarlos. La evolución producida supone llegar, por último, al marketing colaborativo, que se sustenta en un nuevo individuo que, mediante las relaciones, ayuda a la organización a crear valor y a atraer y fidelizar nuevos consumidores (Serrano-Cobos, 2016; García-Pascual,Alguacil \& Molina-García 2020). Supone asumir las perspectivas de la NTE de Pérez-Gónzalez y Massoni (2009), así como la metodología derivada de la misma propuesta por Tur-Viñes y Monserrat-Gauchi (2014) y complementada por Segarra-Saavedra, Martínez-Sala y Monserrat-Gauchi (2018). Supone contemplar al consumidor en el centro de la estrategia de comunicación por su papel como agente y medio social colaborativo, participativo e interactivo (Cortés, 2009). De esta manera, las marcas deben perder el miedo a los posibles comentarios negativos de los usuarios y aceptar que las marcas son también del consumidor y son construidas por éste (Martínez-Sala, Monserrat-Gauchi \& Campillo-Alhama, 2017). Las EDN no pueden ignorar hoy día al consumidor o a sus públicos, ya que es innegable el valor y credibilidad que tienen los contenidos generados por los usuarios (CGU) (Alonso-González, 2015).

El paradigma del marketing y la comunicación experiencial que sustenta el establecimiento de relaciones -entre consumidores y públicos y las marcas/instituciones- en las experiencias vividas con éstas directamente o a través de otros individuos, no puede ser dejado de lado por organizaciones que precisamente promueven el deporte y las experiencias derivadas de su práctica. De igual modo, es reseñable la importancia de conocer y segmentar a los diferentes públicos de las entidades deportivas (Haro-González, Pérez-Ordas, Leyton-Román, Caamaño-Guadix \& Nuviala, 2018).

La gestión de la comunicación organizacional en las Entidades Deportivas Náuticas

Sin duda, la irrupción del medio on line en el escenario comunicativo ha supuesto un punto de inflexión en el estudio de la comunicación organizacional (Vilaplana-Aparicio, et al., 2017; Caffarel-Serra, Ortega-Mohedano \& Gaitán-Moya, 2017; Simancas-González \& García-López, 2017; Túñez-López, 2012). La gestión de la comunicación en las organizaciones deportivas náuticas, en concreto, ha sido analizada por diferentes autores desde esta perspectiva (de-Moragas, 2007; de-Pablos- Heredero, 2003; Fernández-Peña \& RamajoHernández, 2014).

En general, los autores analizados destacan el escaso aprovechamiento de las entidades deportivas españolas en cuanto a las posibilidades del medio on line y la escasa planificación estratégica y relacional de la comunicación en este tipo de organizaciones (Olabe-Sánchez, 2009; Celaya, 2011; Sotelo-González, 2012; Fernández-Souto et al., 2019).

Salinero-López (2014) apuesta por la necesidad de fomentar el diseño de estrategias 2.0 y propiciar la interacción con los seguidores de eventos y marcas deportivas. El autor diferencia el comportamiento seguido por las entidades deportivas en España comparándolo con Estados Unidos, donde sí se realiza una planificación de la estrategia de comunicación potenciando los valores de marca y actuando de manera integral en medios y soportes.

Las posibilidades de internet suponen un desafío a las entidades deportivas (Llopis-Goig, 2016) afectando también a los canales de distribución, ya que posibilitan el emitir competiciones deportivas en directo por este medio. Este es 
un hecho constatado (David \& Millward, 2015) que las entidades deportivas deberían aprovechar. No en balde, Paché e Ika (2017) resaltan las posibilidades que poseen el marketing y las relaciones públicas en la comunicación de los equipos deportivos.

Las EDN además de con el público en general, por su naturaleza estructural están obligadas a mantener una comunicación constante con sus socios, y por otro lado, dado el carácter asociativo de este tipo de organizaciones, también con las asociaciones en las que se integran (OlabeSánchez, 2009).

En los siguientes epígrafes de este artículo constataremos si las EDN han sido conscientes de estas posibilidades y las han asumido en el seno de sus organizaciones. Esto supondría planificar su estrategia de comunicación en base a ellas (Carrillo-Durán, 2016), o puede que por el contrario, sigan ancladas en la concepción de que las principales labores del gabinete de comunicación de una entidad deportiva son: la función periodística, la función de relaciones públicas y, las funciones de marketing y publicidad (VázquezBurgos, 2004).

\section{Material y Método}

Tras el análisis y revisión bibliográfica realizada, los autores se plantean las siguientes preguntas de investigación:

PI.1- Las EDN no gestionan su comunicación desde una perspectiva estratégica: la percepción de la planificación y gestión de la estrategia de la comunicación ha quedado desfasada. Precisa de una revisión y actualización acorde al panorama actual de medios y públicos.

PI.2- Las EDN no desarrollan un Plan Estratégico de Comunicación (PEC) para la gestión relacional con sus públicos.

PI.3- La formación de los recursos humanos responsables de la labor de comunicación en las EDN no es adecuada para gestionar de forma relacional y estratégica la comunicación de las mismas.

PI.4- Las EDN desarrollan una comunicación relacional convencional, aunque sí existe planificación en la comunicación para difundir sus eventos/actividades a los sus públicos: socios, asociaciones en las que se integran y público en general.

Como consecuencia de las preguntas de investigación planteadas, se derivan los siguientes objetivos de investigación que es necesario acometer:

O.1- Conocer la gestión de comunicación llevada a cabo en las EDN.

O.2- Determinar la existencia del departamento de comunicación en las EDN, su funcionamiento y la formación de su personal.

O.3- Conocer si las EDN realizan un PEC con periodicidad determinada.

O.4- Conocer si la comunicación con sus steakholders o públicos de interés (socios, asociaciones y públicos en general) se realiza desde un planteamiento estratégico.

O.5- En base a la información recabada, plantear un modelo de planificación válido, desde las teorías estratégicas de comunicación y de gestión relacional, para este tipo de organizaciones que contemple a sus diferentes públicos.

\section{Sistema de recogida de información y universo de es-} tudio

Tras la pertinente revisión bibliográfica, se ha llevado a cabo una observación de campo u observación participante. Esta supone contemplar sistemática y detenidamente cómo se desarrolla la situación que queremos estudiar, sin manipularla ni modificarla, tal cual es (Duverger, 1996; RuizOlabuénaga, 2012).

En este caso se eligió el Real Club de Regatas de Alicante, que forma parte de la Real Federación Española de Vela. El periodo de tiempo ha sido de un año. Durante el mismo, se investigó el modelo de comunicación empleado en esta entidad, para aplicar los resultados de este modelo al diseño de un cuestionario on line que pudiera ser auto-administrado. Una vez diseñado y validado, el cuestionario ha sido enviado a todas las entidades que conforman el universo de estudio: 164 Entidades Deportivas Náuticas registradas en la página web de la Real Federación Española de Vela.

De las 164 entidades que representan el universo de estudio, se ha obtenido respuesta del $48.7 \%$ de ellas. Se ha utilizado la encuesta general y auto-administrada (Cea, 1996) formada por 33 preguntas, la mayoría cerradas. El tipo de muestreo empleado es de conveniencia; no probabilístico, ya que ha consistido en seleccionar el 100\% de los individuos a investigar.

Los resultados a continuación presentados, suponen un reflejo de la realidad de la gestión de la comunicación en este tipo de organizaciones. Los autores, además, proponen un modelo de plan estratégico para la gestión de la misma en la EDN.

\section{Resultados}

En primer lugar, se hace necesario analizar la existencia de un departamento propio de comunicación en las EDN, éste sería el encargado de diseñar un plan estratégico para gestionar la comunicación relacional con los públicos de este tipo de organizaciones.

Los resultados de la encuesta arrojan que sólo el 37\% de las 164 entidades analizadas posee un departamento interno para gestionar la comunicación del club con sus públicos. La mayoría (63\%) no valora como necesario la creación de este departamento. Más adelante, se analizará si este tipo de entidades subcontrata de manera externa la función de planificación y gestión de la comunicación.

Respecto a la formación de sus trabajadores, del 37\% de

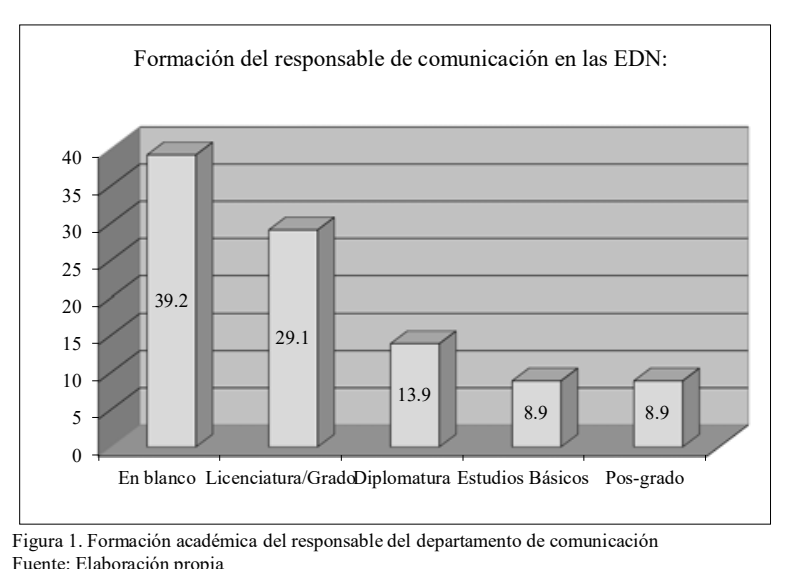


entidades que sí posee departamento de comunicación, casi un $40 \%$ de las mismas no ha indicado la formación o nivel de estudios de su responsable. De entre el $60 \%$ que sí responde, destaca el 29\% que ha indicado que tiene una formación de grado o licenciatura, un 14\% una diplomatura, y 9\% un pos-grado o estudios básicos. Si consideramos válidos los estudios de: Licenciatura/Grado, Diplomatura y Pos-grado, podemos afirmar que un $44 \%$ de las EDN con departamento de comunicación poseería personal válido al frente del mismo. Esto sin conocer si la formación de este personal es en un ámbito relacionado con comunicación.

Otro dato interesante, es conocer quién asume, planifica y desarrolla las funciones de comunicación en las organizaciones deportivas náuticas. Estos datos pueden observarse en la Figura 2.

En las EDN que no poseen un departamento propio de comunicación, es decir el 63\% de las mismas, personas de otros departamentos asumen las funciones de comunicación en el 58\% de los casos. En un $11.4 \%$ de estas entidades, las labores de comunicación no son tenidas en cuenta o asumidas por nadie en particular y, sólo en el $9 \%$ de las entidades, se sub-contrata a una agencia especializada para la gestión de comunicación.

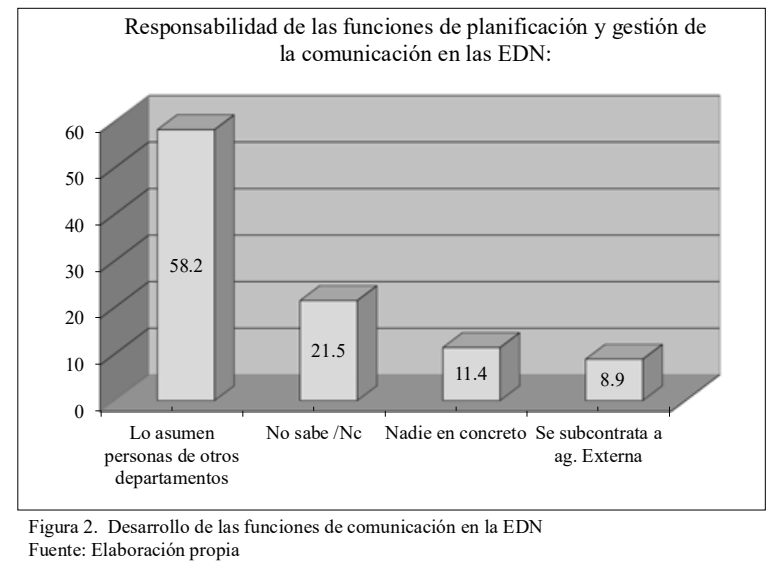

Respecto a la elaboración de un plan estratégico de comunicación (PEC), sólo un 19\% de las EDN analizadas desarrollan un PEC. El 81\% de este tipo de organizaciones no planifica estratégicamente la comunicación o, como hemos analizado en anteriores resultados, no dedican recursos humanos a la planificación y gestión de su comunicación.

Respecto a las variables analizadas referidas a la gestión relacional de la comunicación en las EDN, se han identificado tres públicos clave: los socios de estas organizaciones, las asociaciones en las que se integran estas entidades y, los públicos en general. Pasamos a comentar las variables analizadas relativas a la comunicación relacional con cada uno de estos públicos que podrán observarse en la Figura 3.

Cabe destacar que la mayoría de EDN persiguen el objetivo de informar a sus socios y movilizarlos en lo referido a las actividades programadas por la propia entidad (91\% de las EDN). En segundo lugar este tipo de organizaciones deportivas tiene como objetivo difundir eventos, iniciativas o actividades relacionadas con el deporte (80\%). Llama la atención que los dos objetivos mayoritarios de las EDN en relación a sus socios hagan referencia a dar a conocer o comunicar. En este sentido, actúa también el tercer objetivo mayori- tario, perseguido por un $65 \%$ de las organizaciones: trasladar los valores propios a sus socios a través de sus medios de comunicación. En el apartado Discusión se retomarán las aportaciones de diferentes autores relativas al poder del público en la generación de contenidos y valor sobre la marca.

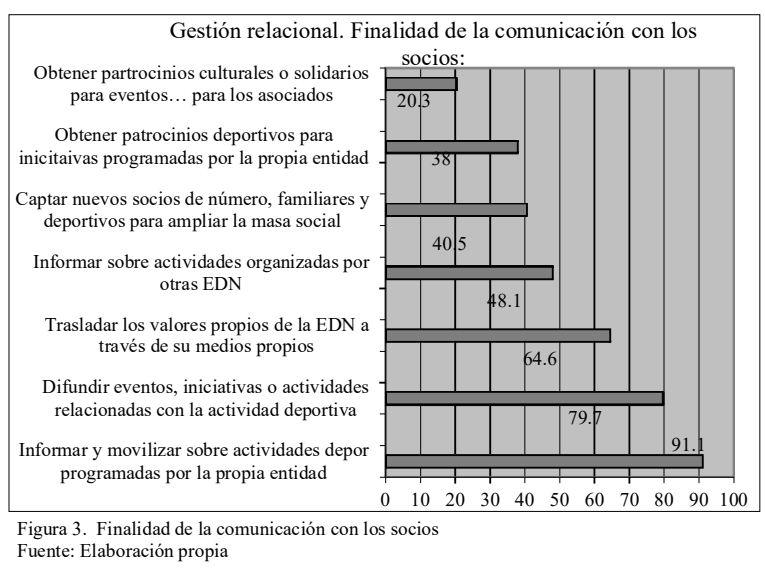

Respecto a la relación de las EDN con asociaciones del sector deportivo, cabe destacar que el $84.8 \%$ de las EDN están asociadas a la Real Federación Española de Vela, y un 3.8\% lo están en la Federación Española de Asociaciones de Puertos Deportivos y Turísticos. Además, una amplia mayoría de ellas afirma estar integrada en las asociaciones autonómicas o regionales de vela de su ámbito geográfico. Por ello, resulta necesario conocer la comunicación que se establece entre estas asociaciones y los miembros que las integran, es decir, las EDN estudiadas. Según los datos que recoge la Figura 4, la mayoría de organizaciones deportivas náuticas manifiesta que las asociaciones de las que forma parte llevan a cabo reuniones con el objetivo de planificar sus acciones, eventos, el funcionamiento y la comunicación entre sus asociados. En total, el 73.4\% de las EDN manifiesta que las asociaciones establecen reuniones de planificación estratégica de forma anual (21.5\%); semestral (20.3\%); o trimestral (31.6\%). Sólo el 5\% de las EDN manifiesta no reunirse nunca con las asociaciones de las que forma parte con el objetivo de planificar sus actuaciones. Y un $21.5 \%$ de las mismas afirma hacerlo esporádicamente, sin periodicidad fija.

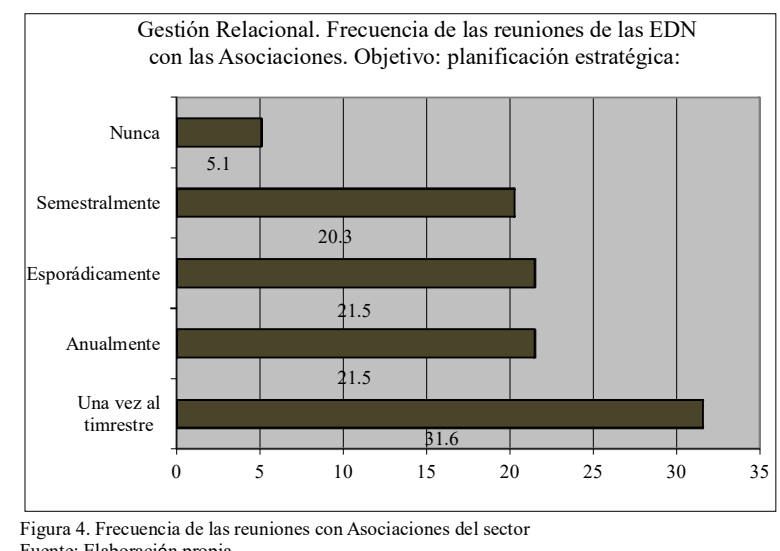

Fuente: Elaboración propia

La gestión relacional de la comunicación en las EDN pasa por planificar la comunicación con el público en general al que estas entidades se dirigen. Normalmente las EDN organizan eventos o competiciones deportivas que suelen estar 
abiertas a la sociedad, ciudad, comarca o entorno geográfico en el que están ubicadas. Respecto a la responsabilidad de la organización y difusión de este tipo de actividades, casi la mitad de ellas (45.6\%) manifiesta que se organizan, ejecutan y comunican por parte de personas con otras funciones diferentes a las del departamento de comunicación de la EDN. Tal como puede verse en la Figura 5, el 43\% de las entidades manifiesta realizarlo desde el propio departamento, y una minoría lo realiza conjuntamente con una agencia externa en colaboración con su departamento (10\%), o bien delegando totalmente esta función a la empresa externa $(1.3 \%)$.

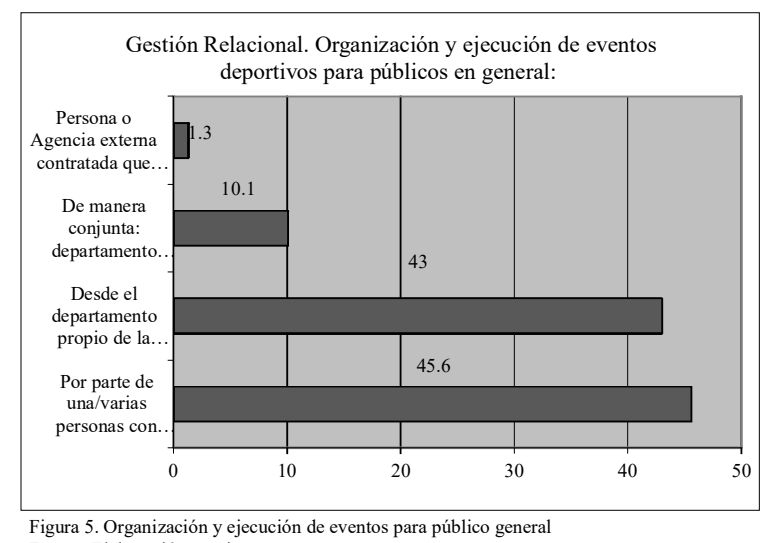

Fuente: Elaboración propia

A la vista de los resultados presentados, podemos afirmar que la mayoría de EDN no planifican de manera estratégica y relacional su comunicación. Es más, este tipo de organizaciones deportivas, relega la comunicación a un segundo lugar, otorgándole pocos recursos humanos (y por tanto económicos) tanto a su planificación como a la gestión de la misma. Podemos deducir que las actividades de comunicación desarrolladas por este tipo de entidades se desarrollan de manera improvisada, y son gestionadas en la mayoría de ocasiones por profesionales de otros ámbitos, pero a todas luces, inexpertos y no formados en comunicación.

\section{Discusión}

Siguiendo a los principales autores, la función esencial del responsable de comunicación radicaría en elaborar y hacer ejecutar con eficiencia el PEC de la organización deportiva náutica (Pérez-González \& Massoni, 2009; MonserratGauchi \& Sabater-Quinto, 2017; Segarra-Saavedra, et al., 2018; Simancas-González \& García-López, 2017; Carrillo-Durán, 2016). Para lograrlo, este departamento debería participar activamente en el proceso estratégico global de la entidad, siendo un punto importante de referencia y apoyo de la Dirección (Tur-Viñes \& Monserrat-Gauchi, 2014; Túñez-López, 2012). De este modo, se aseguraría el correcto planteamiento de las estrategias, estableciendo con claridad el concepto de identidad del club náutico (visión y misión), conociendo cómo es visto desde dentro y fuera por todos sus públicos y su entorno, así como investigando sobre esa percepción y cómo afecta a los objetivos generales de entidad la imagen que todo ello genera.

Así pues para que la estructura de comunicación de un club náutico responda al modelo estratégico de comunica- ción y gestión relacional que plantean los autores citados, se deben tener presente las distintas áreas susceptibles de generar comunicación en una EDN. Del responsable de comunicación deberían depender todas las áreas de comunicación de club que son las que establecen relaciones con sus públicos de interés o steakholders. De este modo, siguiendo a Pérez-Gónzalez y Massoni (2009), entendemos que para desarrollar este modelo de PEC en las EDN se requiere de una serie de procesos en los que se manifieste la estructura del modelo y los públicos a los que va dirigido. La implementación del modelo de comunicación que se propone, vendría condicionado por una fase previa de análisis de la situación existente, en cuanto a la estructura comunicativa de la entidad para así establecer una serie de estrategias de comunicación, que conlleven las metas que se pretende alcanzar.

La propuesta de modelo de PEC y gestión relacional para las organizaciones deportivas náuticas, tal y como puede observarse en la Figura 6, considera como públicos clave de un club: los socios, las asociaciones en las que se integran y la sociedad de su entorno. Esta es una de las peculiaridades de este tipo de organizaciones que no encontraríamos en otras empresas o instituciones: el carácter asociativo de su génesis y de la estructura asociativa externa en la que se integran. Gracias a ellos, este tipo de entidades pervive y promueve la actividad náutica deportiva. Por este motivo, los clubes náuticos deberían reflexionar profundamente sobre la situación externa e interna de su organización, el papel de sus públicos y de la comunicación con ellos.

La Junta Directiva de una organización náutica debería marcar una serie de objetivos (Simancas-González \& GarcíaLópez, 2017; Tur-Viñes \& Monserrat-Gauchi, 2014; TúñezLópez, 2012) que la Dirección intentará alcanzar mediante una serie de estrategias diseñadas, planificadas y ejecutadas por el responsable de comunicación, en unos plazos definidos por la Dirección. De este modo, el responsable de comunicación sería quien gestionaría toda comunicación de una EDN mediante un proceso de comunicación integral, con el objetivo de lograr una imagen positiva y de confianza que ayude a alcanzar prestigio, reputación u otros objetivos corporativos definidos previamente.

Para ello, los clubes náuticos se deberían plantear una serie de fases o procesos como los propuestos en el Modelo de la Figura 6 (pre-diagnóstico: análisis de la situación, diagnóstico; definición de objetivos; elección de estrategias; ejecución: acciones de comunicación; y evaluación: analizar resultados) que ayuden readaptar las estrategias futuras comunicativas-relacionales y organizacionales. Por supuesto, atendiendo a las peculiaridades de este tipo de asociaciones a las que ya se ha hecho referencia. Este modelo planteado surge de las bases o postulados de los autores de la NTE $\mathrm{y}$, además, de la información recabada tanto de la encuesta a las EDN como del trabajo de campo llevado a cabo durante un año en una de ellas. De ahí que sea necesario hacer especial hincapié en el carácter asociativo de este tipo de entidades y que el diseño del modelo haya tenido en cuenta esta serie de particularidades. Sería necesario un desarrollo posterior con un mayor nivel de detalle del modelo que escapa a las posibilidades de esta investigación y que se acometerá en futuras investigaciones. 


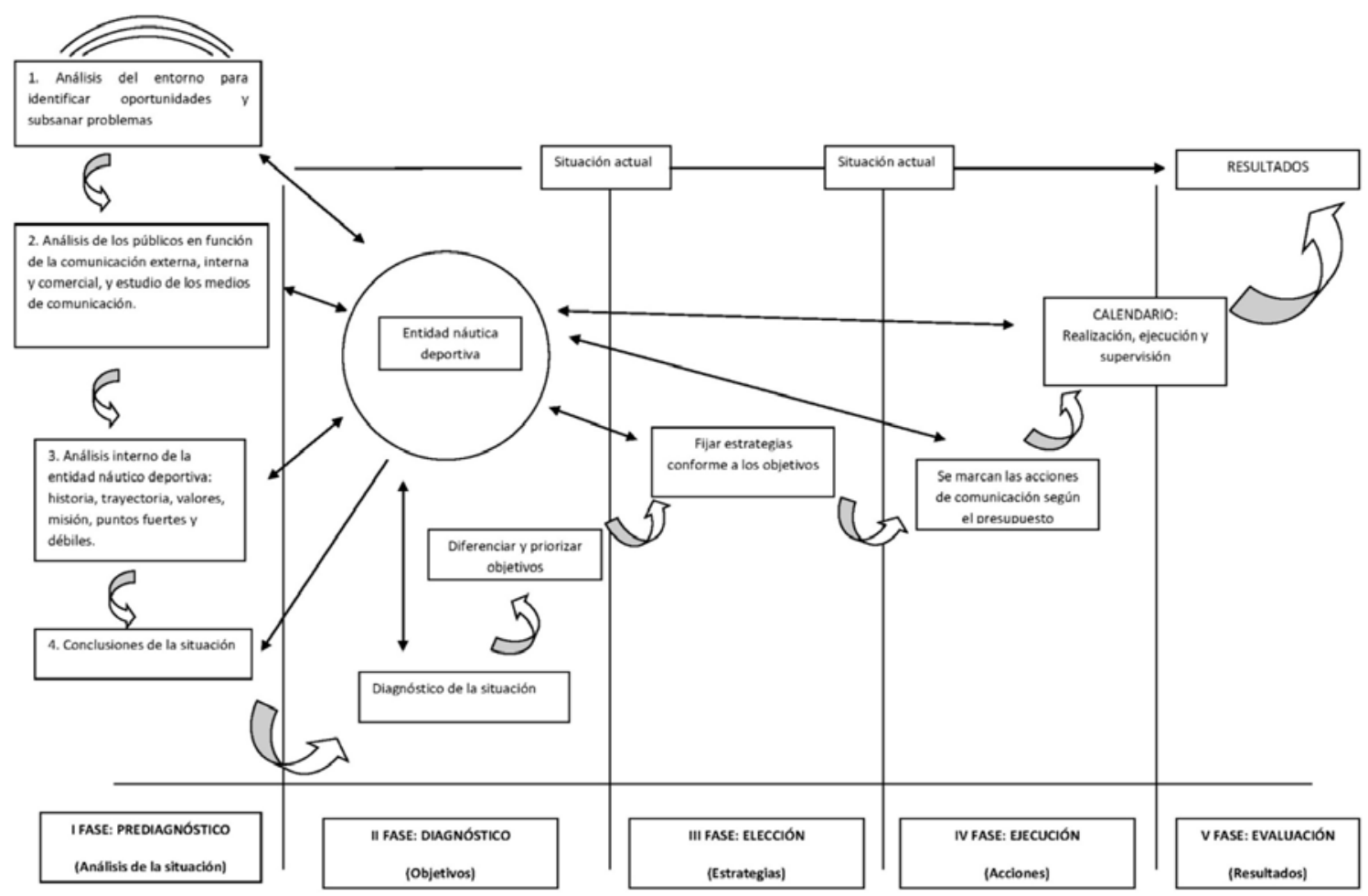

Figura 6. Propuesta de modelo de planificación estratégica y relacional de la comunicación para las EDN Fuente: Elaboración propia a partir de: modelo Estrategar de Pérez-González y Massoni (2009); Tur-Viñes y Monserrat-Gauchi (2014); Monserrat-Gauchi y Sabater-Quinto (2017); SegarraSaavedra, et al. (2018).

\section{Conclusiones}

Según hemos visto en el apartado anterior, las EDN no gestionan su comunicación desde una perspectiva estratégica y relacional. (Valida la pregunta de investigación 1, PI.1)

Por tanto, puede afirmarse que la gestión de la comunicación que realiza este tipo de organizaciones deportivas es anacrónica y precisa de una actualización desde el panorama actual de las teorías estratégicas de comunicación.

La mayoría de EDN no desarrolla un PEC para la gestión relacional con sus públicos. (Valida la pregunta de investigación 2, PI.2). Por tanto, los objetivos que pueden perseguir estas organizaciones no se están alcanzando de forma óptima. No se implica a sus públicos en la comunicación y creación de imagen de las EDN (Masip, et al., 2019), en su filosofía y necesidades, ni se les convierte en prosumers (MartínezSala, et al., 2018). De este modo se desaprovechan las ventajas derivadas de una correcta planificación estratégica que revierten en que sean los propios públicos los co-creadores de valor para este tipo de entidades.

La formación de los recursos humanos al frente de la planificación y la gestión comunicativa y relacional de las EDN no es adecuada. (Valida la pregunta de investigación 3, PI.3). Según Olabe-Sánchez (2009) y Celaya (2011) con la llegada de las nuevas tecnologías de la información y comunicación, los clubes náuticos han creado portales webs para gestionar la imagen y el prestigio de la propia entidad, unidos a blogs, boletines digitales y redes sociales, entre otros. Si la interacción con sus públicos a través de este medio y de estos soportes no se planifica desde una perspectiva estra- tégica y relacional, tal y como hemos visto que sucede, la comunicación queda al amparo de la improvisación y la imagen y los objetivos de las organizaciones deportivas náuticas pueden verse perjudicados.

Las EDN sí planifican, si quiera mínimamente, la difusión de las actividades que desarrollan, tanto a las asociaciones en las que se integran, como al público en general y a sus socios. (Valida la pregunta de investigación 4, P.I.4). El medio Internet ha proporcionado la posibilidad a las EDN de comunicarse de forma rápida con los socios, con el público en general y con otras asociaciones y empresas (de-Moragas, 2007). De este modo, para mantener una relación de comunicación activa entre sus públicos, el responsable del departamento de comunicación del club náutico tiene que acomodar sus discursos, noticias o comunicaciones a esta nueva idiosincrasia y características de los social media (Caldevilla, 2010).

Esta forma de difundir sus eventos a sus públicos, sería correcta sí se planificara una estrategia relacional de comunicación desde una visión holística de la misma (Pérez-González \& Massoni, 2009) y llevando a cabo una planificación estratégica integral en la que se contemplen todos los públicos sobre los que actuar, así como los medios y canales en los que es posible hacerlo (Monserrat-Gauchi \& Sabater-Quinto, 2017; Segarra-Saavedra, et al., 2018)

El medio internet y las herramientas de comunicación 2.0 han hecho mucho más accesibles las EDN y los eventos deportivos que generan a los públicos de las mismas. No obstante, estas entidades no han comprendido el enorme potencial de las nuevas herramientas de comunicación que, 
planificadas y gestionadas desde una perspectiva estratégica y relacional, propiciarían una mayor participación social de sus públicos de manera online en la vida de los clubes náuticos, un rápido alcance de los objetivos propuestos y un mayor conocimiento y reconocimiento social a su labor.

\section{Referencias}

Alguacil, M., Crespo-Hervás, J. \& Pérez-Campos, C. (2020). Análisis sociodemográfico de la percepción de la marca en un servicio deportivo público: del target al buyer persona. Retos: nuevas tendencias en educación física, deporte y recreación, 37, 139-146

Alonso-González, M. (2015). Las redes sociales como canal de comunicación de las marcas de moda españolas. El caso de Zara, Mango y El Corte Inglés. Index. comunicación: Revista científica en el ámbito de la Comunicación Aplicada, 5(1), 77-105. Recuperado de http:// journals.sfu.ca/indexcomunicacion/index.php/ indexcomunicacion/article/view/177/167

Bauman, Z. (2013). La cultura en el consumo de la modernidad líquida. Madrid, España: Fondo de Cultura Económico.

Caffarel-Serra, C., Ortega-Mohedano, F., \& Gaitán-Moya, J. A. (2017). Investigación en Comunicación en la universidad española en el período 2007-2014. El profesional de la información, 26(2), 218-227. doi: 10.3145/ epi.2017.mar.08

Caldevilla, D. (2010). Nuevas lecturas del concepto de publicidad a partir de las TIC. Questiones publicitarias, 15, 35-51. doi: 10.5565/rev/qp.82

Caro, J. L., Luque, A., \& Zayas, B. (2015). Nuevas tecnologías para la interpretación y promoción de los recursos turísticos culturales. Pasos. Revista de Turismo y Patrimonio cultural, 13(4), 931-945. Recuperado de http:// ojsull.webs.ull.es/index.php/Revista/article/view/778

Carrillo-Durán, M. V. (2016). Importancia del profesional de la información en la dirección de comunicación de las organizaciones. El profesional de la información, 25(2), 272278. doi: 10.3145/epi.2016.mar.14

Castells, M. (2000). La era de la información. Economía, sociedad y cultura. La sociedad red. Madrid, España: Alianza.

Cea, M. Á. (1996). Metodología cuantitativa: estrategias y técnicas de investigación social. Madrid, España: Síntesis.

Celaya, J. (2011). La empresa en la web 2.0: El impacto de las redes sociales y las nuevas formas de comunicación online. Barcelona, España: Gestión 2000.

Cortés, M. (2009). Bienvenido al nuevo marketing. En Sanagustín, E. (Ed.), Del 1.0 al 2.0: Claves para entender el nuevo marketing (pp. 6-23). España: Bubok Publishing.

David, M., \& Millward, P. (2015) Sport and new Media. En Giulianotti, R. (Ed.), Routledge Handbook of the Sociology of Sport. London, England: Routledge

De-Moragas, M. (2007). Comunicación y deporte en la era digital. En IX Congreso de la Asociación Española de Investigación Social Aplicada al Deporte (AEISAD), Las Palmas de Gran Canaria.
De-Pablos-Heredero, C. (2003). Actitudes en la utilización de las tecnologías web para la comunicación en las entidades deportivas. En Taller de trabajo Nuevas Tecnologías aplicadas al Deporte.

Duverger, M. (1996). Métodos de las ciencias sociales. Barcelona, España: Ariel.

Fernández-Peña, E., \& Ramajo-Hernández, N. (2014). La comunicación en el deporte global: los medios y los Juegos Olímpicos de verano (1894-2012). Historia y comunicación social, 19, 703-714. doi: 10.5209/ rev_HICS.2014.v19.45171

Fernández-Souto, A.M., Puentes-Rivera, I. \& Vázquez-Gestal, M. (2019). Gestión de la comunicación en las competiciones Deportivas regulares: Fútbol sala, voleibol, baloncesto y balonmano en España. Retos: nuevas tendencias en educación física, deporte y recreación, 36, 9-16.

García-Orosa, B. (2005). Los altavoces de la actualidad: radiografía de los gabinetes de comunicación. La Coruña, España: Netbiblo.

García-Pascual, F., Alguacil, M. \& Molina-García, N. (2020). Predicción de la lealtad de los usuarios de centros deportivos privados. Variables de gestión y bienestar subjetivo. Retos: nuevas tendencias en educación física, deporte y recreación, 38, 16-19

Haro-González, M., Pérez-Ordas, R., Leyton-Román, M., Caamaño-Guadix,A. \& Nuviala,A. (2018). Grupos de usuarias del mercado deportivo femenino. Retos: nuevas tendencias en educación física, deporte y recreación, 34, 123-127

INE, Instituto Nacional de Estadística, (2018). Indicadores demográficos. Recuperado de http://www.ine.es/dyngs/ INEbase/es/operacion.htm?c=Estadistica_C\&cid $=1$ $254736177003 \&$ menu $=$ ultiDatos\&idp $=1254735573002$

Llopis- Goig, R. (2016). Deporte, medios de comunicación y sociedad. RICYDE. Revista Internacional de Ciencias del Deporte, 12(44), 86-89. doi: 10.5232/ricyde2016.044ed

Marauri-Castillo, Í., Pérez-Dasilva, J., \& Rodríguez-González, M. M. (2015). La búsqueda de la comunidad de marca en las redes sociales. Los casos de Telepizza, Vips y Burger King. Trípodos, 37, 133-149. Recuperado de http:// w w w. t r i p od o s . c o m/i n d e x.ph p / Facultat_Comunicacio_Blanquerna/article/view/256

Martínez-Sala,A. M., \& Campillo-Alhama, C. (2018). El video como soporte en la narrativa digital de los eventos turísticos 2.0. Miguel Hernández Communication Journal, 9,227-260. doi: 10.21134/mhcj.v0i9.230

Martínez-Sala, A. M., Monserrat-Gauchi, J., \& CampilloAlhama, C. (2017). The relational paradigm in the strategies used by destination marketing organizations. Revista Latina de Comunicación Social, 72, 374-396. doi: 10.4185/ RLCS-2017-1170

Martínez-Sala, A. M., Segarra-Saavedra, J., \& MonserratGauchi, J. (2018). Los millennials como prosumers y adprosumers en las redes sociales corporativas. Cuadernos.Info, 43, 137-159. doi: 10.7764/cdi.43.1335

Masip, P., Ruiz-Caballero, C., \& Suau, J. (2019). Active audiences and social discussion on the digital public sphere. Review article. El profesional de la información, 28(2). doi: 10.3145/epi.2019.mar.04

Monserrat-Gauchi, J., \& Sabater-Quinto, F. (2017) Planifica- 
ción estratégica de la comunicación en redes sociales. Barcelona, España: UOC.

Olabe-Sánchez, F. (2009).La comunicación no convencional en los clubes de fútbol. Pensar la publicidad, 3(1), 121137. Recuperado de http://revistas.ucm.es/index.php/ PEPU/article/view/PEPU0909120121A/15348

Olabe-Sánchez, F. (2015). El gabinete de comunicación como impulsor de la gestión comunicativa de los clubes de fútbol. Revista Mediterránea de Comunicación, 6(1), 83-104. doi: 10.14198/MEDCOM2015.6.1.06

Ortiz, J. C. (2010). Shorts/Cortos. Bogotá, Colombia: Ediciones Gamma.

Paché, G., \& Ika, L. (2017). European Professional Football Club Image and Human Capital Management. International Journal of Business Administration and Management Research, 3(2), 30-34.

Pérez-González, R. A., \& Massoni, S. (2009). Hacia una teoría general de la estrategia. Barcelona, España: Ariel.

Ruiz-Olabuénaga, J. I. (2012). Metodología de la investigación cualitativa. Barcelona, España: Deusto.

Salinero-López, J. C. (2014). Nuevas formas de comunicación en el deporte. El twitter de Cristiano Ronaldo. Valladolid, España: Universidad de Valladolid. Recuperado de http://uvadoc.uva.es/bitstream/10324/6252/1/TFGN.157.pdf

Segarra-Saavedra, J., Martínez-Sala, A. M., \& MonserratGauchi, J. (2018). Planificación estratégica de la comunicación integrada. Razón y Palabra, 22(3-102), 152-178. Recuperado de http://www.revistarazonypalabra.org/ index.php/ryp/article/view/1263

Serrano-Cobos, J. (2016). Tendencias tecnológicas en internet: hacia un cambio de paradigma. El profesional de la información, 25(6), 843-850. doi: 10.3145/ epi.2016.nov.01
Simancas-González, E., \& García-López, M. (2017). Gestión de la comunicación en las universidades públicas españolas. El profesional de la información, 26(4), 735-744. doi: 10.3145/epi.2017.jul.17

Solana, D. (2010). Postpublicidad. Reflexiones sobre una nueva cultura publicitaria en la era digital. Barcelona, España: DoubleYou.

Soler-Adillon, J., Sora, C., Freixa, P., \& Ribas, J. I. (2016). Perfil del profesional de la comunicación interactiva: fundamentos, actualidad y perspectivas. El profesional de la información, 25(2), 196-208. doi: 10.3145/epi.2016.mar.06

Sotelo-González, J. (2012). Deporte y social media: el caso de la Primera División del fútbol español. Historia y Comunicación Social, 17, 217-230. doi: 10.5209/ rev_HICS.2012.v17.40607

Stelzner, M. (2017). Social media marketing industry report 2017. Social Media Examiner.

Túñez-López, M. (2012). La gestión de la comunicación en las organizaciones. Zamora, España: Comunicación Social.

Tur-Viñes, V., \& Monserrat-Gauchi, J. (2014). El plan estratégico de comunicación. Estructura y funciones. Razón y palabra, 3(88). Recuperado de http:// www.razonypalabra.org.mx/N/N $88 /$ Varia/ 46_VinesGauchi_V88.pdf

Vázquez-Burgos, M. Á. (2004). El profesional de las relaciones externas: los gabinetes de comunicación desde la perspectiva periodística. Barcelona, España: Editorial Bosch.

Vilaplana-Aparicio, M. J., Iglesias-García, M., \& MartínLlaguno, M. (2018). Communication of innovation through online media. El profesional de la información, 27(4), 840-848. doi: 10.3145/epi.2018.jul.13

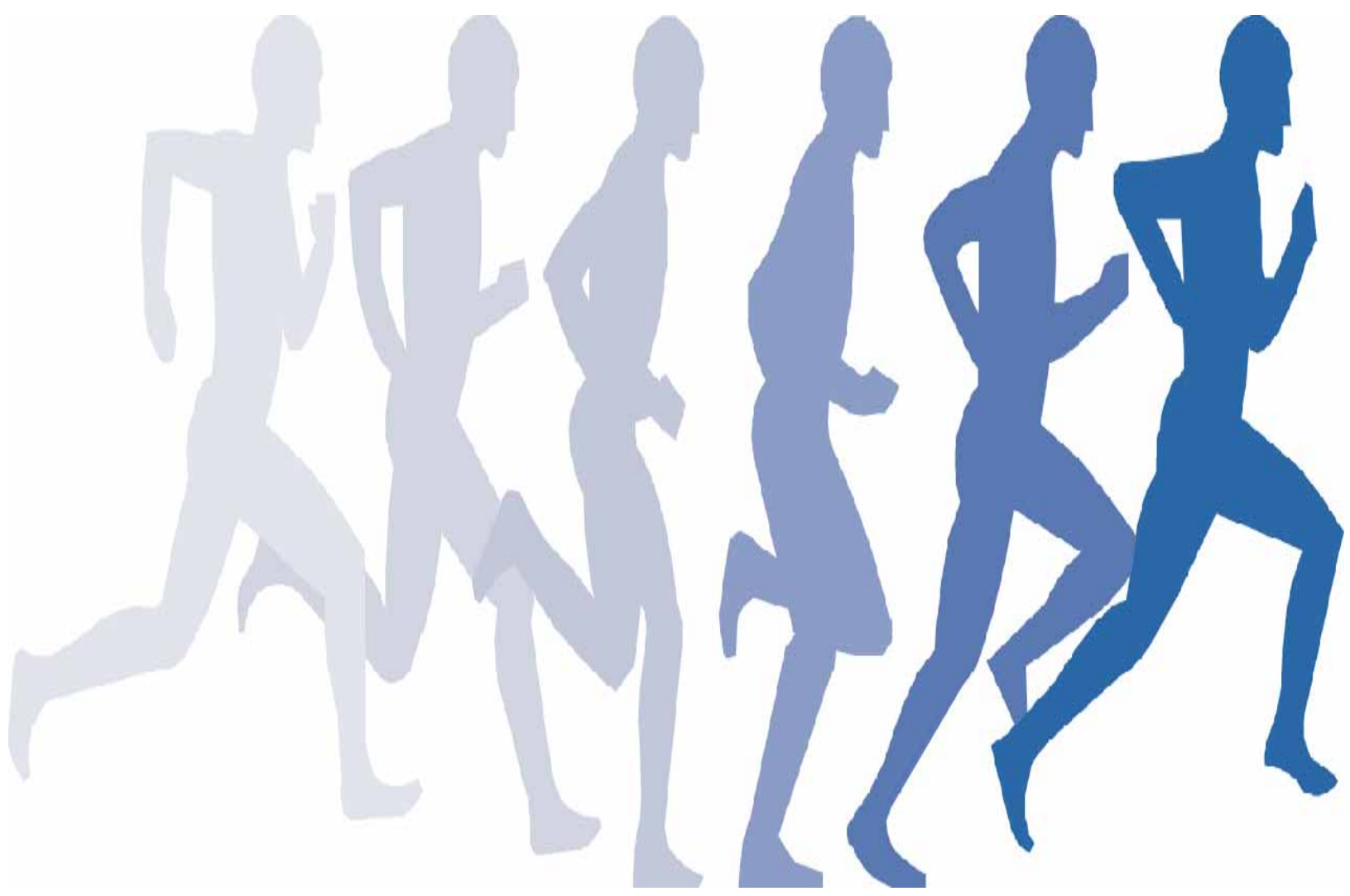

